\title{
Criação e avaliação de um objeto de aprendizagem para a pegada ecológica
}

\author{
Mauro Barreto $^{1}$, Felipe Torres ${ }^{1}$, Gabrielly Silva $^{1}$, Lucas Mascena ${ }^{1}$, \\ Raucy Dantas ${ }^{1}$, Adriana Damasceno ${ }^{1}$ \\ ${ }^{1}$ Centro de Ciencia e Tecnologia Agroalimentar - UFCG \\ Rua Jario Vieira Feitosa - 58.840-000 - Pombal - PB - Brazil \\ \{mauro.jnior7, felipe.alves.torres7, adriana.damasceno\}@gmail.com
}

\begin{abstract}
Nowadays, we use computers in many areas. However, there is a lack of usage for computational resources in the classroom. Computers aid the conscious needs for better management of natural resources. This work presents a learning object for students from high schools in order to illustrate the ecological footprint. We used the Scratch software to implement the learning object. Also, 63 students performed an evaluation by using the Bloom taxonomy. Results show that the evaluation was successful.
\end{abstract}

Resumo. Os computadores são usados em diversas áreas. No entanto, a utilização de recursos computacionais em sala de aula é escassa, principalmente em escolas públicas. Há uma necessidade de conscientização do uso dos recursos do planeta e um dos meios de aprendizado desta teoria pode ocorrer por meio dos computadores. Este trabalho apresenta um objeto de aprendizagem que ilustra o conceito de pegada ecológica direcionado para alunos de ensino médio. Usamos a linguagem Scratch e o formato de quiz. Sua avaliação foi realizada através de questionários por 63 alunos de ensino médio com o auxílio da taxonomia de Bloom. Os resultados mostraram aceitação por ambos os públicos.

\section{Introdução}

Os computadores fornecem inúmeras contribuições para a sociedade, incluindo áreas como contabilidade, engenharia, medicina e educação. Particularmente na área da educação, o destaque é notório, já que o ensino a distância, assim como a leitura e a pesquisa de diversos tipos de dados são permitidos através do uso do computador.

Paralelamente, há necessidade de conscientização do uso dos recursos naturais do planeta [Tietenberg and Lewis 2016]. Uma das formas de medir os impactos que o estilo de vida de um indivíduo tem sobre a natureza é a pegada ecológica. Este é um cálculo que representa um resumo do modo de vida das pessoas. Como análise resultante dessa compilação, é obtida a estimativa de quantos planetas Terra seriam necessários para abrigar a população humana, caso todos no planeta mantivessem os mesmos hábitos de consumo da pessoa cujos dados foram analisados [Dias 2015].

Adicionado a esta realidade, há um desuso dos computadores para fins didáticos em escolas públicas [Damasceno et al. 2016]. Os alunos e professores têm acesso a computadores e smartphones, mas não os utilizam durante o processo de aprendizado. Com 
VIII Congresso Brasileiro de Informática na Educação (CBIE 2019)

Anais do XXV Workshop de Informática na Escola (WIE 2019)

isso, é necessário que existam programas dedicados a ilustrar conceitos explorados em sala de aula. Umas das formas de incentivar o uso dos computadores neste contexto são os objetos de aprendizagem [Wiley 2002].

Este trabalho apresenta um objeto de aprendizagem sobre a pegada ecológica. $\mathrm{O}$ programa foi implementado por alunos do curso de Engenharia Ambiental do Campus de Pombal da Universidade Federal de Campina Grande usando a linguagem Scratch e foi avaliado por 63 alunos da rede estadual de ensino. Como resultados, pôde-se observar boa aceitação do objeto com pontos de melhoria relacionados à acessibilidade e usabilidade.

\section{Referencial Teórico}

Scratch [Marji 2014] é uma linguagem de programação visual desenvolvida pelo Massachusetts Institute of Technology (MIT). Ela é constituída de blocos gráficos que representam comandos clássicos de linguagens de programação convencionais [Marji 2014].

Os Objetos de Aprendizagem (OA) são unidades informacionais reutilizáveis que ilustram conceitos usando sistemas computacionais. Segundo o Learning Objects Metadata Workgroup [LOM 2018], eles são “qualquer entidade, digital ou não digital, que possa ser utilizada, reutilizada ou referenciada durante o aprendizado suportado por tecnologias". Sua principal característica é a reusabilidade facilitada pelo uso de repositórios que os catalogam por tema, nível de dificuldade, assunto e relação com outros objetos [Audino and da Silva Nascimento 2010].

Uma forma de avaliar a eficácia do objeto de aprendizagem é utilizar a taxonomia de Bloom. Ela é uma taxonomia específica para objetivos de processos educacionais dividida entre os domínios cognitivo, afetivo e psicomotor, sendo estruturada em níveis de complexidade crescente. Sendo assim, a estrutura do processo cognitivo da taxonomia de Bloom é dividida em seis níveis: lembrar, entender, aplicar, analisar, avaliar e criar [Ferraz and Belhot 2010].

Vários trabalhos com resultados sobre objetos de aprendizagem foram reportados na literatura, mas nenhum envolveu a taxonomia de Bloom como método de avaliação com foco na criação de um objeto de aprendizagem para ilustrar o conceito de pegada ecológica. A Tabela 1 mostra características sobre alguns objetos desenvolvidos, a saber: autor, assunto, software usado e informações sobre o público avaliado.

Tabela 1. Trabalhos relacionados

\begin{tabular}{|l|l|l|l|}
\hline Autor & Assunto & Software & Público avaliado \\
\hline \hline [Silva et al. 2014] & $\begin{array}{l}\text { Saneamento } \\
\text { básico }\end{array}$ & Scratch & $\begin{array}{l}\text { 5 professores e } \\
\text { alunos do ensino } \\
\text { básico }\end{array}$ \\
\hline [Andrade and Rodrigues 2016] & Estatística & Flash & $\begin{array}{l}16 \text { alunos de esco- } \\
\text { las públicas }\end{array}$ \\
\hline [da Silva and Costa 2017] & Matemática & $\begin{array}{l}\text { Ferramentas de } \\
\text { bancos de objetos } \\
\text { de aprendizagem }\end{array}$ & $\begin{array}{l}\text { 40 alunos de } \\
\text { graduação }\end{array}$ \\
\hline [Vania and Vieira 2016] & Microbiologia & $\begin{array}{l}\text { Flash, edição de } \\
\text { audio e de vídeo }\end{array}$ & $\begin{array}{l}\text { Alunos } \\
\text { graduação de }\end{array}$ \\
\hline
\end{tabular}


VIII Congresso Brasileiro de Informática na Educação (CBIE 2019)

Anais do XXV Workshop de Informática na Escola (WIE 2019)

\section{Métodos}

Utilizamos o objeto de aprendizagem por dois anos dentro de um projeto cujo objetivo foi ofertar cursos sobre o pensamento computacional em uma universidade pública local. $\mathrm{O}$ público-alvo foi composto por 63 alunos do ensino médio de escolas públicas da cidade de Pombal e São Bentinho que participaram dos cursos. O objeto de aprendizagem foi criado em três etapas: (i) escolha e análise do tema, (ii) implementação e (iii) aplicação e avaliação.

$\mathrm{Na}$ escolha e projeto do tema, os tutores do projeto propuseram diversos assuntos que poderiam ser usados para a implementação do OA. Como a maioria estudava Engenharia Ambiental e os impactos ambientais são assuntos de bastante relevância tanto para os tutores quanto para os alunos do projeto, optou-se pelo tema pegada ecológica. Também foi ideia dos tutores usar o formato de quiz para a implementação do OA conforme a calculadora da pegada ecológica da WFF Brasil [PEG 2018].

$\mathrm{Na}$ fase de implementação, os tutores usaram a linguagem de programação Scratch. A escolha desta ferramenta se deu pela familiaridade dos tutores, já que este foi o software usado para oferecer os cursos sobre o pensamento computacional durante dois anos. Além disso, os recursos gráficos oferecidos por ela permitem estimular a atenção dos alunos.

Após o uso do objeto de aprendizagem, os alunos responderam o survey de 29 questões. O objetivo foi identificar o nível de entendimento dos alunos com relação aos conceitos de pegada ecológica usando os critérios de avaliação propostos pela taxonomia de Bloom. Para isso, as questões foram mapeadas de acordo com os itens da taxonomia usando a seguinte ordem: lembrar (1-2), entender (3-5), aplicar (6-8), analisar (9-11), avaliar (12-18), criar (19-21) e afetivo (22-28).

\section{Resultados}

O objeto de aprendizagem foi implementado usando a linguagem de programação Scratch e é executado em qualquer computador. O personagem Homer Simpson é usado para orientar o usuário sobre que ações devem ser tomadas para que o OA seja executado com sucesso, conforme mostram as Figuras 1 e 2. Em termos gerais, as telas são divididas em três grupos: (i) apresentação, (ii) perguntas e (iii) pegada ecológica do indivíduo ${ }^{1}$.

Com o objetivo de melhorar a atratividade das telas para os alunos, usamos desenhos dos Simpsons retratando situações similares ao assunto das 15 perguntas que compõem o jogo (Figura 1). Na sequencia, um conjunto de 4 respostas possíveis é mostrado. Dependendo da resposta escolhida pelo usuário, pontos são atribuídos à pontuação geral. A pegada ecológica do indivíduo é mostrada na tela final do OA. Os mesmos intervalos de valores mostrados na calculadora da pegada ecológica da WWF Brasil são usados para determinar quantos planetas seriam necessários para sustentar os hábitos de consumo do usuário. A Figura 2 mostra um exemplo de tela final do OA.

Os resultados da avaliação do objeto de aprendizagem foram medidos sob a visão dos alunos. O questionário pós-teste ofereceu respostas em escala de 1 a 5 e foi aplicado para 63 alunos de escolas públicas. Mais de $50 \%$ dos respondentes informaram em mais

\footnotetext{
${ }^{1} \mathrm{O}$ objeto está disponível no link https://goo.gl/Qk6z66 para ser livremente baixado
} 
VIII Congresso Brasileiro de Informática na Educação (CBIE 2019)

Anais do XXV Workshop de Informática na Escola (WIE 2019)

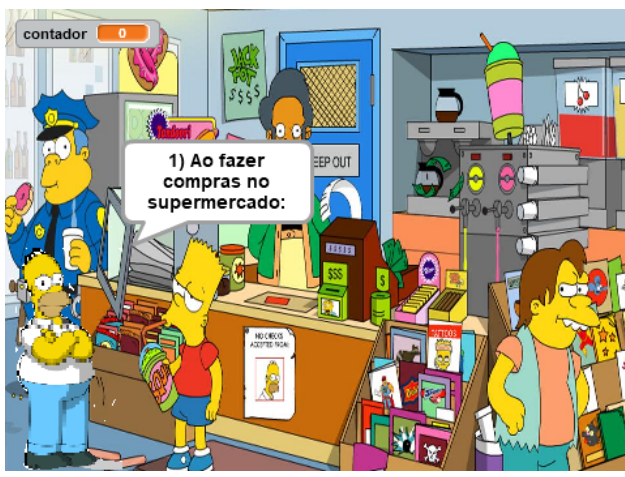

Figura 1. Segunda tela do $O A$

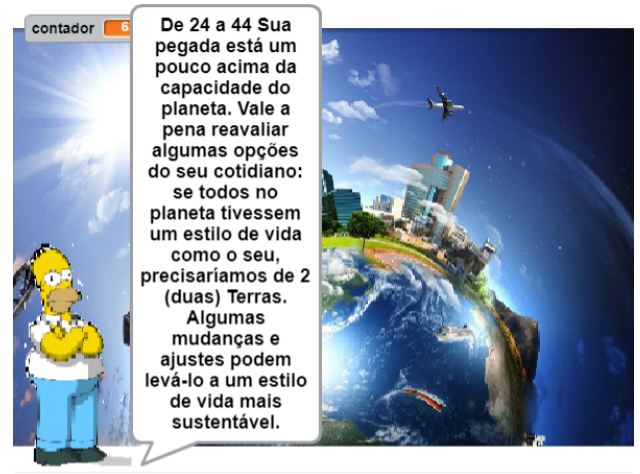

Figura 2. Tela final do $O A$

de $71 \%$ das questões os níveis 4 ou 5, indicando grau elevado de satisfação com relação ao objeto de aprendizagem. Ademais, quando agrupamos as respostas 4 e 5 de cada questão, as respostas de acordo com as questões da taxonomia de Bloom e realizamos uma média aritmética destes valores, tem-se os seguintes achados: lembrar (69\%), entender $(73,57 \%)$, aplicar $(54,8 \%)$, analisar $(50,17 \%)$, avaliar $(75,04 \%)$, criar $(54,76 \%)$ e afetivo $(64,17 \%)$. Por conseguinte, observamos que as habilidades mais pontuadas foram avaliar e entender, enquanto que os itens menos favorecidos são aplicar e analisar.

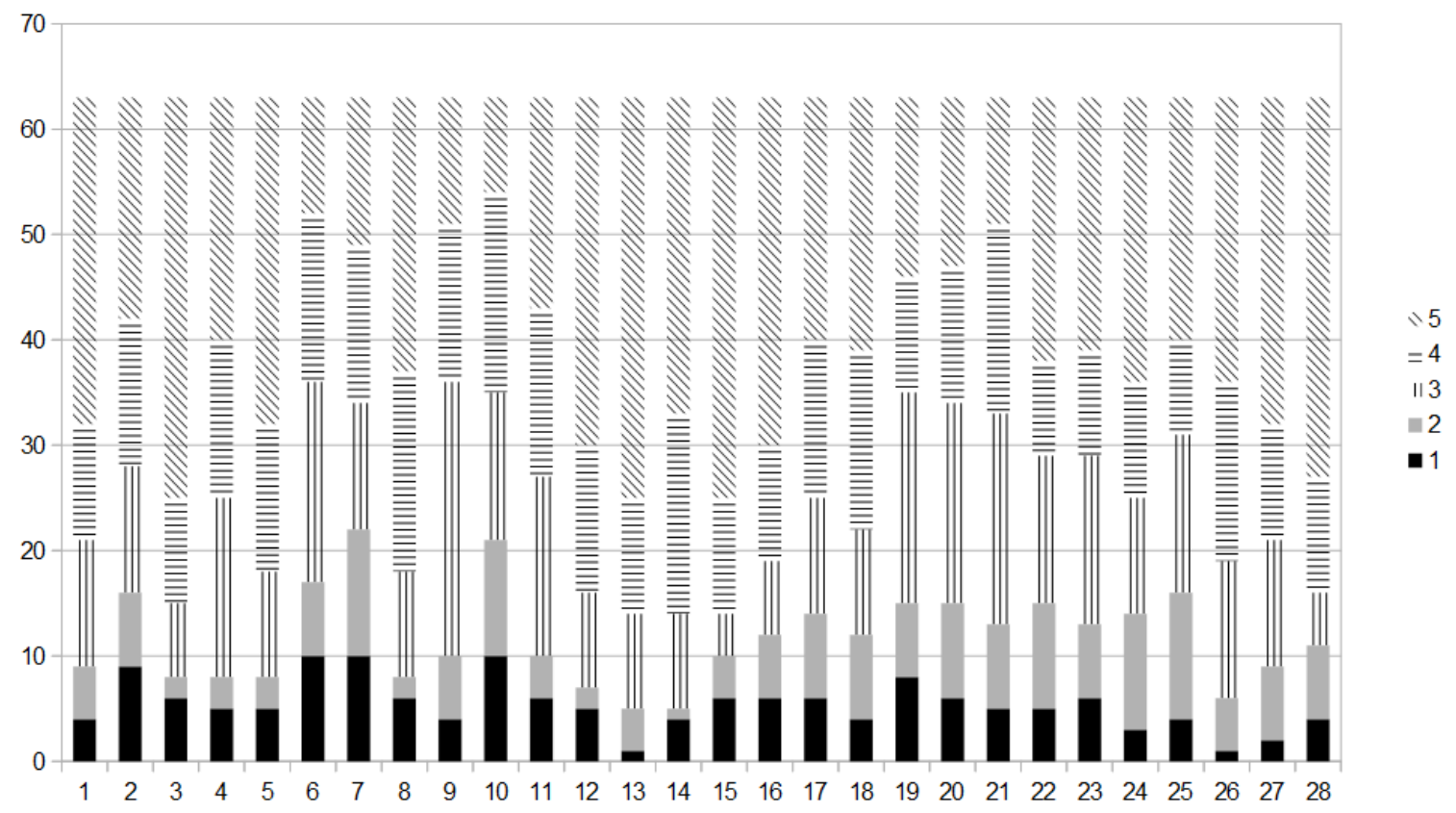

Figura 3. Gráfico de avaliação pós-teste

\section{Conclusões}

Este trabalho apresentou um objeto de aprendizagem cujo objetivo é ilustrar o conceito de pegada ecológica. O objeto foi aplicado para 63 alunos do ensino médio de escolas públicas e avaliado por alunos. Depois da aplicação do objeto, a maioria dos respondentes indicou alto grau de satisfação do aprendizado sobre o tema. Um questionário foi aplicado como base na taxonomia de Bloom para aferição da qualidade do objeto. 
VIII Congresso Brasileiro de Informática na Educação (CBIE 2019)

Anais do XXV Workshop de Informática na Escola (WIE 2019)

Dentre as contribuições deste trabalho, estão a apresentação de um método para avaliação de objetos de aprendizagem que inclui a taxonomia de Bloom e questionários avaliativos do objeto com a apresentação do conteúdo de pegada ecológica. Os trabalhos futuros incluem o uso do objeto em outros contextos como o ensino superior e professores de escolas públicas.

\section{Referências}

(2018). Calculadora da pegada ecologica. http: / / www . pegadaecologica . org . br/. Accessado: 09-06-2018.

(2018). Learning objects metadata workgroup. https://standards. ieee.org/ develop/wg/LOMWG12 . html. Accessado: 09-06-2018.

Andrade, G. D. O. and Rodrigues, C. (2016). Vem Aprender: Objetos de Aprendizagem para o ensino de Estatística. Anais do Workshop de Informática na Escola, 22(1):602.

Audino, D. F. and da Silva Nascimento, R. (2010). Objetos de aprendizagem-diálogos entre conceitos e uma nova proposição aplicada à educação. Revista Contemporânea de Educação, 5(10).

da Silva, K. C. and Costa, M. N. D. (2017). Jogos digitais na escola : a utilização como objetos de aprendizagem no ensino da matemática. In Anais do $23^{\circ}$ Workshop de Informática na Escola, pages 21-30.

Damasceno, A. C., Lopes, M., Andrade, R., Nobrega, S., and Almeida, I. (2016). Descrevendo o uso dos computadores nas escolas públicas da Paraíba. Revista Brasileira de Informática na Educação, 24(3):47-61.

Dias, G. F. (2015). Pegada ecológica e sustentabilidade humana. Global Editora e Distribuidora Ltda.

Ferraz, A. P. D. C. M. and Belhot, R. V. (2010). Taxonomia de Bloom: revisão teórica e apresentação das adequações do instrumento para definição de objetivos instrucionais. Gestão \& Produção, 17(2):421-431.

Gasque, K. C. G. D. (2016). Objetos de Aprendizagem para o Letramento Informacional. Revista Ibero-Americana de Ciência da Informação, 9(2):387-405.

Marji, M. (2014). Learn to Program with Scratch: A Visual Introduction to Programming with Games, Art, Science, and Math. No Starch Press.

Silva, A. M. d. S., Moraes, D. A. S. d. S., and Batista, S. C. F. (2014). Objetos de Aprendizagem em Scratch para Estudo de Saneamento Básico: Percepções de Alunos e Professores. In Workshop de Informática na Educaçao (WIE), pages 370-379.

Tietenberg, T. H. and Lewis, L. (2016). Environmental and natural resource economics. Routledge.

Vania, K. and Vieira, M. (2016). Objeto de aprendizagem empregado como recurso multimídia na microbiologia. Teorias e práticas em tecnologias educacionais, pages 123149.

Wiley, D. (2002). Learning objects need instructional design theory. The ASTD eLearning handbook, pages 115-126. 